\title{
Barriers and facilitators to implementing coronary care networks in South Africa: a qualitative study
}

\author{
Willem Stassen ${ }^{1,2}$, Lisa Kurland ${ }^{1,3}$, Lee Wallis ${ }^{2,4}$, Maaret Castren $^{5}$, Craig Vincent-Lambert ${ }^{6}$
}

1. Department of Clinical Research and Education, Karolinska Institutet.

2. Division of Emergency Medicine, Stellenbosch University.

3. School of Medical Sciences, Orebro University.

4. Division of Emergency Medicine, University of Cape Town.

5. Department of Emergency Medicine and Services, Helsinki University.

6. Department of Emergency Medical Care, University of Johannesburg.

\begin{abstract}
Background: ST-elevation myocardial infarction (STEMI) is on the rise in sub-Saharan Africa. South Africa consistently fails to deliver timely reperfusion to these patients, possibly due to under-developed coronary care networks (CCN).

Objectives: To determine the current perceived state of CCNs, to determine the barriers to optimising CCNs and to suggest facilitators to optimising CCNs within the South African context.

Methods: A qualitative descriptive approach was employed, by performing two structured in-depth and two focus group interviews ( $\mathrm{n}=4$ and 5, respectively), inviting a purposely heterogeneous sample of 11 paramedics $(\mathrm{n}=4)$, doctors $(\mathrm{n}=5)$, and nurses $(\mathrm{n}=2)$ working within different settings in South African CCNs. Recorded interviews were transcribed verbatim and subjected to content analysis.
\end{abstract}

Results: Participants described an under-resourced, unprioritised and fragmented CCN with significant variation in performance. Barriers to CCN optimisation resided in recognition and diagnosis of STEMI, transport and treatment decisions, and delays. Participants suggested that thrombolysing all STEMI patients could facilitate earlier reperfusion and that pre-hospital thrombolysis should be considered. Participants highlighted the need for regionalised STEMI guidelines, and the need for further research.

Conclusion: Numerous barriers were highlighted. Healthcare policy-makers should prioritise the development of CCNs that is underpinned by evidence and that is contextualised to each specific region within the South African health care system.

Keywords: Health care system, emergency care, cardiovascular disease.

DOI: https://dx.doi.org/10.4314/ahs.v20i1.39

Cite as: Stassen W, Kurland L, Wallis L, Castren M, Vincent-Lambert C. Barriers and facilitators to implementing coronary care networks in South Africa: a qualitative study. Afri Health Sci. 2020;20(1):338-50. bttps:/ / dx.doi.org/10.4314/abs.v20i1.39

\section{Introduction}

Cardiovascular disease (CVD) is an emerging epidemic in sub-Saharan Africa and other low- to middle-income countries such as South Africa. ${ }^{1}$ It is estimated that mortality due to ischaemic heart disease (IHD) will increase by approximately $70 \%$ in African men and women by $2030 .^{1}$

A consequence of IHD is myocardial infarction, and ST-elevation myocardial infarction (STEMI). STEMI is a time-sensitive emergency that requires emergent treatment to reperfuse the ischaemic myocardium and in so doing, limit the extent of permanently infarcted tissue. ${ }^{2}$ International guidelines recommend that reperfusion primarily takes place via percutaneous coronary intervention (PCI) within 90 minutes of STEMI diagnosis. ${ }^{3}$ Failing PCI-access, recommendations suggest that thrombolyt-

willem.stassen@uct.ac.za

(C) 2020 Stassen W et al. Licensee African Health Sciences. This is an Open Access article distributed under the terms of the Creative commons Attribution License (https://creativecommons.org/licenses/BY/4.0), which permits unrestricted use, distribution, and reproduction in any medium, provided the original work is properly cited. 
ic therapy be administered within 10 minutes of STEMI diagnosis. ${ }^{3}$

South African publications show difficulties ensuring reperfusion within recommend timeframes. ${ }^{4-6}$ For patients who underwent PCI, only $61.3 \%$ of patients received stents within 24 hours and $18 \%$ of patients received a thrombolytic upon admission. ${ }^{4}$ In another study set in Cape Town, South Africa, the median time to thrombolytic therapy was 54 minutes. ${ }^{5}$ Finally, in Pretoria, South Africa only $3 \%$ of eligible patients received thrombolytic therapy within 60 minutes. ${ }^{6}$ South Africa also has a tremendous shortage in PCI-capable facilities, ${ }^{7}$ and is characterised by poor access to PCI based on geographic and socio-economic status. ${ }^{7}$

In order to achieve reperfusion time targets, the European Society of Cardiology recommends that a coronary care network, defined as "the implementation of networks between hospitals with various levels of technology, linked by a prioritised and efficient ambulance service" be established. ${ }^{3}$ These networks should aim at optimising care while minimising delays, focusing on an integrated and coordinated approach.

It has been suggested that shortfalls in honouring reperfusion targets may be related to system delays within these coronary care networks. ${ }^{3,6}$

Currently, there is a paucity of available literature on the state of coronary care networks within low- to middle-income countries such as South Africa. A lack of information in this area remains a barrier to the implementation and improvement of these networks. To this end, we sought to answer three questions through the perceptions of our participants who currently work within the South African coronary care network. These questions were: 1) What is the current perceived state of coronary care networks within the South African context? 2) What are some of the barriers to optimising coronary care networks that may deliver reperfusion strategies within the time frames described by international guidelines? 3) What are some suggested facilitators to optimising a coronary care network?

\section{Methods}

Using purposeful sampling and a constructivist methodological orientation, this exploratory study employed a qualitative research method, and made use of two individual, in-depth and two focus group interviews. This study is reported according to the COnsolidated criteria for REporting Qualitative research (COREQ) guidelines. ${ }^{8}$

\section{Reflexivity}

All interviews were conducted by the principal author (PA). The principal author is a male Emergency Care Practitioner (ECP) with 7 years' experience within the South African prehospital context in road, aeromedical and mobile intensive care units. At the time, the PA was a $\mathrm{PhD}$ student with formal training in qualitative research, and interview techniques. Primarily based within the City of Johannesburg of the Gauteng province, he has experience of other South African provinces and the greater African continent secondary to aeromedical retrieval experience. The interest of the PA in this particular research topic related to seeing immense delays in reperfusion. Relationships between the PA and the participants had been established previously during clinical work or routine patient care or further during attendance of conferences in emergency care or cardiology. Participants understood the reasons for undertaking research within this particular field, as they had heard the PA previously talk on similar topics during conference proceedings.

\section{Setting}

At the time of the study, the North West and Northern Cape provinces had no emergency and timely access to PCI-capable facilities. Variable access to these facilities within the Western Cape and Gauteng provinces were appreciable. South Africa has a tremendous shortage in PCI-capable facilities, ${ }^{7}$ have significant delays to reperfu$\operatorname{sion}^{4-6}$ and is characterised by poor access to PCI based on geography and socio-economic status. ${ }^{7}$

\section{Data collection procedure}

Using a constructivist orientation, qualitative data collection was completed between June and October 2017. All in-depth and focus group interviews were conducted in private venues where data collection could commence without the presence of non-participants and interruption, at times convenient for the participants. The first in-depth interview was conducted at a home office, the first focus group interview was conducted at the specific private hospital's training boardroom, and the second focus group interview was conducted in a boardroom at the South African Emergency Medicine Conference in October 2017. The final in-depth interview was conducted at the cardiologist's private consulting rooms. All interviews were conducted in English, except for the first focus group interview, which was conducted in Afrikaans and English. 
An interview schedule was developed using current literature on the barriers to the optimisation of coronary care networks, as well as specific contextual insight by the author's clinical experience. An initial in-depth interview was conducted to develop and refine the interview schedule and to gather ideas that were planned to be explored further during focus group interviews. Data from this interview were included as answers were consistent and the discussion schedule did not change significantly. This is further an acceptable practice as qualitative research is progressive in intent, each interview building on a subsequent data collection encounter. ${ }^{9}$

The discussion schedule firstly addressed what participants felt the current state of CCNs in South Africa was. From here, prompts and probes aimed to elucidate specific data of each participants' perception and experiences of barriers within their own setting and solutions that they might consider worth implementing to facilitate the development of effective CCNs.

Two focus group interviews (with four and five participants respectively) were then completed to gain an integrated view of the CCNs of South Africa with people who would normally be expected to work together.

Participants were approached by the PA via telephone call or face-to-face and invited to participate in the study. Participants were selected from the prehospital and in-hospital context of both state and private healthcare settings with variable PCI access, and from various geographic locales. It was expressly aimed to interview participants working in areas of both PCI-capable facilities and those who do not have immediate PCI access. By sampling participants from differing backgrounds, it was felt that many views from different lenses could be obtained in this initial, exploratory study. The qualitative approach of focus group interviews was selected in order to generate insights into the different perspectives between individuals from different settings, thus making results more representative of the South African context. ${ }^{10}$ The demographic profile of the participants is shown in Table 1. These stakeholders would have sufficient experience and knowledge to unpack the problem of implementing and optimising coronary care networks within the South African context and provide appropriate content that could lead to meaningful analysis.

Table 1 Demographic profile of participants

\begin{tabular}{|c|c|c|c|c|}
\hline Participant & Position & Province of work & Sector of work & PCl Access \\
\hline $\mathrm{P} 1$ & $\mathrm{ECP}$ & Gauteng & Private & Yes \\
\hline $\mathrm{P} 2$ & RN: ICU & North West & Private & No \\
\hline P3 & RN: EC & North West & Private & No \\
\hline P4 & ALS & North West & Private & No \\
\hline P5 & EC Doctor & North West & Private & No \\
\hline P6 & ECP & Western Cape & State & No \\
\hline P7 & EP & Gauteng & State & Yes \\
\hline P8 & EP & Gauteng & State & No \\
\hline P9 & EP & Northern Cape & State & No \\
\hline P10 & ECP & Gauteng & Variable & Variable \\
\hline P11 & Cardiologist & $\begin{array}{l}\text { Gauteng and } \\
\text { Mpumalanga }\end{array}$ & State and Private & Yes \\
\hline
\end{tabular}

During the focus group interviews, many aspects related to the role of the cardiologist surfaced. It was therefore decided to triangulate ${ }^{11}$ the results by conducting a depth interview with an interventional cardiologist who has experience in both state and private healthcare sectors. Hereafter, it was felt that data saturation had occurred, and interviews were concluded. Data saturation was defined as the point where new data tended to be redun- dant, and the same comments from participants were heard repeatedly. ${ }^{12}$

Ethical approval for this study was granted by the $\mathrm{Hu}-$ man Research Ethics Committee of the University of Stellenbosch (N16/10/120). Written informed consent was obtained from all participants. Particular consent was sought from each participant for the audio recording of 
the qualitative interviews. Participants were also permitted to withdraw from the study at any time prior to transcription, as each individual participant would no longer be identifiable hereafter. Participants in the focus groups were also requested to treat the opinions of other participants in confidence and with respect. Anonymity was maintained throughout the data collection procedure and identifying data has been removed and is not reported on. The interviews were self-transcribed, and identifying data were not recorded on the transcriptions. Audio recordings were deleted after data analysis. Further confidentiality was ensured by limiting data access to the research team.

\section{Data analysis}

With specific permission, all interviews were recorded in duplicate via iPhone 6S (Apple Inc. California, United States) Voice Memo Application (iOS 11) and H1 Handy Audio Recorder (ZOOM Corporation, Tokyo, Japan).
Hereafter, recordings were downloaded onto a secured computer and transcribed verbatim by the PA. All identifying data were omitted from the transcripts, which were stored under password protection.

Transcripts were subjected to descriptive content analysis by the PA, after immersion in the data. ${ }^{13,14}$ Using Atlas. ti (Scientific Software Development GmbH; Berlin, Germany) specific meaning units were condensed, coded and categorised. Codes were further corroborated by the senior author. Analysis was completed to the manifest level as the aim was to describe phenomenon rather than ascribe meaning to the data. ${ }^{15}$ Analysis was further completed utilising a deductive-dominant approach by considering literature and existing theories through reflexivity and the discussion schedule. ${ }^{16}$ During immersion in the data, as new categories emerged, these were developed inductively. ${ }^{16}$ Later, further deductive approaches interrogated these new categories throughout the transcribed dataset, allowing for recoding. ${ }^{16}$ Development is exemplified in Table 2.

Table 2 Categorical development using qualitative content analysis

\begin{tabular}{lll}
\hline Meaning Unit & Code & Category \\
\hline "So in that particular setting the number of & Limited CCU beds & There are many resource \\
coronary care unit beds is so miniscule" & & constraints \\
"most GPs don't know what an ECG is, they don't ECG Interpretation & $\begin{array}{l}\text { Poor recognition and } \\
\text { know how to interpret it," }\end{array}$ & $\begin{array}{l}\text { diagnosis } \\
\text { "we can't adjust the system based on no data." }\end{array}$ \\
\hline
\end{tabular}

\section{Trustworthiness}

The trustworthiness of data presented within this qualitative approach was ensured by considering aspects of credibility, transferability, dependability and confirmability. ${ }^{11}$

Credibility was ensured by employing qualitative methods that are well-established; by making use of a discussion schedule consistently applied by the same interviewer; by triangulation (the use of both focus groups and individual depth interviews); by using participants who freely volunteered their time, thus ensuring that participants were genuinely interested in giving data freely (promotes honesty); and by examining the results in the context of previous research findings for congruence, as presented in this report. ${ }^{11}$

As this was a descriptive qualitative work with a small sample size, transferability is difficult to ascertain. However, by selecting a heterogenous sample, broad perceptions from different participants could be explored. Furthermore, a thorough (but suitably anonymised) description of the participants and the South African context may provide opportunity for the reader to establish transferability to their context on an individual basis. ${ }^{11}$

Dependability is ensured by providing a description of the data collection procedures and the specific discussion schedule utilised. Further method error was mitigated by ensuring that only the PA completed all interviews, thereby interview techniques should have been similar for all interviews. $^{11}$

To ensure confirmability, the transcribed research was checked by the other authors. Further to this, reflective admission of the PA's own beliefs were considered before and after each interview. Furthermore, data was triangulated between two methods (focus groups and individual 
depth interviews), and corroborated by previous research findings. ${ }^{11}$

\section{Results}

Thirteen participants were approached, and eleven participants were interviewed. Two participants (one from each focus group) were unable to attend on the day of data collection. Inclusion of participants was diverse (Table 1). Interviews lasted between 50 and 77 minutes (average 65.5 minutes). Despite a diverse range of participants, common categories (summarised in Table 3) emerged universally.

Table 3 Emerging categories following content analysis

\begin{tabular}{l} 
1. What is the current perceived state of coronary care networks within the South African \\
context? \\
Increasing patient numbers, with more lifestyle diseases \\
Non-communicable diseases are not prioritised \\
The network is characterised by considerable variation in performance \\
The network is perceived to be fragmented \\
There are many resource constraints \\
\hline 2. What are some of the barriers to optimising coronary care networks that may deliver \\
reperfusion strategies within the time frames described by international guidelines? \\
\hline Poor recognition and diagnosis \\
Inappropriate transport decisions \\
Inefficiencies at patient triage \\
There are considerable delays in reperfusion \\
\hline 3. What are some suggested facilitators to optimising a coronary care network? \\
Prehospital Thrombolysis \\
Locally appropriate guidelines \\
Improved clinical governance \\
Research is needed
\end{tabular}

\section{Discussion}

Participants were asked to provide their perceptions on the current state of CCNs in South Africa, what some of the barriers were to optimising CCNs and what they felt would facilitate the optimisation and overcome these barriers. Next, each of the categories (Table 3) that emerged are discussed in turn. In support of this discussion, specific quotations (italicised) extracted from the interviews, are referred to.

\section{Increasing patient numbers, with more lifestyle dis-} eases

1. What is the current perceived state of coronary care networks within the South African context?

After content analysis, five categories related to the current perceived state of CCNs in South Africa emerged. These categories were: increasing patient numbers especially of non-communicable diseases which are not prioritised by policy-makers, and with considerable variation and fragmentation in the network. Finally, the $\mathrm{CCN}$ is perceived to experience many resource constraints.
The epidemiological transition towards non-communicable and lifestyle diseases (such as myocardial infarction) within South Africa ${ }^{17}$ was acknowledged however, it was felt that despite this the overall burden of disease is increasing "we are getting a lot more lifestyle disease, but the infectious burden has not gone away. So its not like thre is less of trauma and less of infectious diseases and replaced by MI, that burden is still the same (P5, ECP)." "So the patient burden is actually compounding in total (P9, EP)." This could have dire consequences to a healthcare system that is already overstretched and under-resourced. ${ }^{18}$

This disease burden was felt to further be underestimated due to poor reporting, "It's not documented in South Africa how many are dying (P11, Cardiologist)." A paucity of health surveillance data as it relates to myocardial infarction has previously been highlighted. ${ }^{19}$ This paucity of epidemiological data could be as a result of misdiagnosis of AMI, thus fuelling a belief that AMI has poor prevalence in (South) Africa, and thus not being prioritised by policy-makers. ${ }^{19}$

African Health Sciences Vol 20 Issue 1, March, 2020 


\section{Non-communicable diseases are not prioritised}

Participants echoed this belief that policy-makers did not place CVD as a priority, "Non-communicable diseases are just relegated to the dustbin (P11, cardiologist)." Policy-makers were criticised by focusing marketing campaigns on HIV and TB, when CVD is equally preventable, "People market $H I V$ very heavily and its perceived to be this big thing, whether or not it is, but its overshadowing something that is preventable (P1, ECP)," but carries similar morbidity in the economically active population, "they cause an enormous amount of morbidity, but they don't seem to be a big priority (P1, EP)." This seems to stem from a belief that CVD is primarily a "Western" affliction and that cardiovascular risk factors are virtually non-existent in the African populous. ${ }^{1}$ Authors warn that this belief is likely seated in absence of evidence, ${ }^{1,19}$ and highlights the need for epidemiology research to mobilise policy-makers into action.

Participants felt that prevention was key to avoiding AMI, "STEMIs could be prevented (P1, ECP)." However, it was felt that policy-makers were not directing priority to prevention strategies, "We are not focusing on preventative medicine (P1, ECP)." This may lead to patients seeking emergency care and presenting with advanced disease and AMI.

\section{The network is characterised by considerable varia- tion}

Once a patient suffers myocardial infarction, there seems to be variation in the way that the patient will access the $\mathrm{CCN}$, and how their diagnosis and treatment is expedited, "every province is like a different country (P2, ECP)." These variations seem to be related to two factors: the initial presenting hospital and the time of day.

Participants expressed experience with variation in care based on whether a patient presents to a clinic, non-PCI-capable or PCI-capable hospital. Presenting to a clinic may be associated with significant delays in diagnosis as they lack the basic resources to make a diagnosis of STEMI, requiring secondary transfer "not even ECGs at the clinics (P8, EP)." The ability to recognise and diagnose STEMI is lacking within these primary care settings, "main issue is... to be able to diagnose the problem (P11, cardiologist)."

Assuming a patient presents to a hospital that does have the ability to diagnose STEMI, emergency centre staff are often not comfortable with making the diagnosis ("If they get to a casualty, we've got the most junior staff in the unit," "they don't know how to interpret it," "people don't know how to diagnose STEMIs" - P11, cardiologist) or thrombolys- ing STEMI, "casualty doctors are scared of giving [thrombo] lytics(P11, cardiologist)." This could likely be due to unrealistic risk-benefit ratio, "it's a risky thing to do (P4, ALS)." This notion is corroborated by a South Africa study that found that only $36 \%$ of STEMIs received a thrombolytic on admission, despite only $1 \%$ of patients having a contraindication for thrombolytic therapy. ${ }^{4}$

Presenting to PCI-Hospitals may see the patient receiving a 12 lead ECG immediately, "At triage we have a doctor, and every chest pain patient gets an ECG at triage, that's checked by a doctor (P7, EP)." However, to receive such an expedited response "it really depends on when you have your heart attack (P7, EP)." "If you manage to arrive... between 08:00 and 16:00 on a weekday, your chances of getting to cath lab immediately are really good, if you arrive outside of those hours, much less good (P7, EP)." Time to fibrinolytic therapy and PCI have been shown to be influenced by whether patients present during working hours or not. Presenting after hours has been associated with prolonged time to receiving reperfusion. ${ }^{5,20}$ Patients who present after hours also have an associated higher mortality. ${ }^{20}$ Time of day variation does not simply relate to what happens inside the facility, but can also affect the access that patients have to healthcare when experiencing signs of AMI, "They can't get there, either taxis are expensive or taxis don't run after hours (P9, EP)."

Healthcare access varies greatly in South Africa, and patients without medical insurance and those who reside in rural areas have the most difficulty with accessing emergency care. ${ }^{21,22}$ Constitutionally, every South African has the right to Emergency Care and no one may be refused emergency stabilisation regardless of their ability to pay. ${ }^{23}$

Expressing their frustration, participants criticised the private healthcare sector for limiting care in patients who are unable to afford healthcare because of "financial decisions, and not patient decisions. And it's a huge private healthcare problem (P5, ECP)." "the biggest frustration of all is in the private sector it all revolves around money (P11, cardiologist)." This seems to be related to the fact that the definition of what "emergency stabilisation," means within the context of myocardial infarction (or STEMI), was "a very vague term (P9, EP)," that was often misinterpreted; "people think if you've given the patient aspirin you've completed emergency care... This is the misperception that needs to change (P11, cardiologist)."

Participants felt that this definition "needs to change. From one where people don't even give a [thrombo]lytic because it costs 
money to one where emergency intervention is part of that framework (P11, cardiology)" because "if an artery is blocked it's an emergency until the artery is reopened (P11, cardiology)." In this manner, the private healthcare sector, with more PCI facilities, ${ }^{7}$ might become more accessible to patients who cannot afford timely reperfusion. Despite this, participants still expressed concern related to cost when patients get treated at private facilities, "who covers the cost is the big question (P11, cardiologist).”

\section{The network is perceived to be fragmented}

Participants felt that the South African CCNs were disjointed, "we've got a very fragmented network (P5, doctor)." This fragmentation seemed to be caused by poor relationships and communication channels between the prehospital providers, emergency centre (EC) staff and cardiology and a "complete lack of cohesion (P11, cardiologist)." This fragmentation may be perpetuated by a lack of trust between the different phases of care. Prehospital providers felt that their prehospital diagnosis was not trusted, "redoing a 12 lead ECG if I present you with a viable 12 lead ECG (P1, ECP)." In turn, EC staff felt that the cardiology often questioned their own judgement, "we thought it still warranted urgent care - cardiology didn't agree (P7, EP)." This was echoed, "Often, there isn't a good working relationship between the people on our end delivering service and the casualty department (P11, cardiologist)." All parties felt that relationships and mutual trust are important aspects of a CCN, "So what you need is a paramedic who knows the casualty doctor, knows the cardiologist and so on..., within that work group..., there's trust (P11, cardiologist)," "We know that from a team point of view the best teams are the ones that work as a cohesive unit (P11, cardiologist)" and argued that consultation would help minimise delays and expedite reperfusion, "if we had a relationship with a hospital or cardiologist group... we could immediately consult with them (P1, ECP)." One way of facilitating trust is through communication. In the context of STEMI, communication can be through ECG telemetry.

Informal telemetry systems exist within the SA CCN, mainly utilising a free messaging app called Whatsapp, "so they send the ECGs to me via Whatsapp and I discuss the ECG with them with the clinical history (P8, EP)." Whatsapp (Whatsapp Inc. California) has been shown to decrease reperfusion times in other settings, internationally. ${ }^{24}$ This modality may also be used to bridge the divide and promote cohesion between teams that are in regional referral networks or from different ambulance services or healthcare sectors, "I created a Whatsapp group for all the paramedics in the area from different services and companies and government (P8, EP)" and could ensure open communication even when other communication infrastructure is not compatible between hospitals and healthcare services.

Another reason for a fragmented and varied $\mathrm{CCN}$, could be a relative lack of resources, that must cope with an increasing patient volume.

\section{There are many resource constraints}

The South African CCN is characterised by low resources, especially in the state healthcare setting. Resource constraints relate to limited healthcare providers, equipment, beds and PCI facilities.

It is recommended that patients who experience symptoms of AMI, should immediately contact EMS for ambulance transport to hospital. ${ }^{3}$ Unfortunately, within the South African setting, ambulance response times are unreliable and protracted. ${ }^{25}$ In the city of Pretoria, South Africa, only $31 \%$ of patients with STEMI are transported to hospital in an ambulance. ${ }^{6}$ Participants felt that this was likely due to the limited ambulance resources, "called an ambulance and it came 4 hours later because they were busy with another call. I definitely think that resources are a problem (P8, EP)" and "most people cannot access timeous EMS services in South Africa (P11, cardiologist)." Such limited resources may lead to delays in secondary transfer to a PCI-capable facility, but may also result in significant prehospital delays to first medical contact and diagnosis.

Assuming an ambulance can reach a patient timeously, guidelines recommend prehospital 12 lead ECG acquisition and STEMI diagnosis on scene. ${ }^{3}$ A certain level of skill and equipment armamentarium is required to make a prehospital diagnosis of STEMI. Participants highlighted the lack of prehospital ECGs, "sometimes there wasn't an ECG monitor for me (P6, ECP)," as well as skilled staff who are able to interpret acquired ECGs for STEMI diagnosis, "I think 80-90\% of our prehospital service is BLS (P9, $\mathrm{EP})$," and "We have very little advanced paramedics (P9, EP)."

Staffing concerns are not isolated to the prehospital environment, and extends to cardiologists, nursing staff and PCI-facility support staff (such as radiology and technicians), "there's less than 200 working interventional cardiologists (P11, cardiologist)." In fact, four out of the nine South African provinces do not have a single cardiologist. ${ }^{26}$ Participants also felt that there are not enough nurses and 
trained PCI-facility support staff, "Then we've got the nursing staff, there is just an absolute paucity of trained cath lab staff which is a massive problem (P11, cardiologist)," and "They don't have sufficient nursing staff, they don't have sufficient radiographers and techs (P11, cardiologist)."

Reasons cited by participants for staff shortages were mainly related to working conditions and remuneration structures, "remuneration is a core issue (P11, cardiologist)," and "Nursing staff didn't get overtime pay (P11, cardiologist)." This lack of incentivisation means that staff are less likely to respond to STEMIs after hours, according to participants, "you're expecting people to get out of bed at midnight... You really need to incentivise people to do that (P11, cardiologist)." Poor remuneration also impacts on staff retention "staff retention is a massive problem (P11, cardiologist)," which may perpetuate poor relationships and a fragmented CCN, "there isn't a good working relationship... One of the big contributors is high turnover (P11, cardiologist)."

Of course, having skilled staff is not the only requirement for timely and accurate STEMI diagnosis. Participants exposed that many clinics or hospitals did not have the ability to obtain a 12 lead ECG, "there are some hospitals without ECGs (P9, EP)," This lack of diagnostic capability could result in significant delays in STEMI diagnosis as secondary transport to other facilities will need to be arranged for, "they will send a patient to the hospital just for an ECG (P8, EP)" with limited EMS resources.

There is a shortage of PCI-capable facilities in South Africa. $^{7}$ Even in facilities with PCI, participants described a lack of emergency access to these facilities due to the limited number of suites, "PCI centres have one cath lab (P11, cardiologist)," which could see a suite busy with elective procedures when an emergent patient presents. Repairs and maintenance to these suites could see the facility being unusable for a protracted period, "they had to replace cath labs and we've just been down for weeks (P11, cardiologist)." Participants mentioned experiences where patients were unable to be treated because of a lack of PCI consumables, "you've run out of wires, catheters or there is no contrast (P11, cardiologist)."

In a system of an increasing burden of cardiovascular diseases, bed shortages for admission of these patients was also pointed out, "the number of coronary care unit beds is so miniscule (P11, cardiologist)." Unfortunately, this bed shortage also blocks access to coronary care for some, "we would turn away...patients' from referring clinicians because we simply don't have the capacity to take them (P11, cardiologist)."

African Health Sciences Vol 20 Issue 1, March, 2020
Barring this obvious challenge of limited resources within the CCN, participants also highlighted a few other barriers.

2. What are some of the barriers to optimising coronary care networks that may deliver reperfusion strategies within the time frames described by international guidelines?

Four main perceived barriers to optimising $\mathrm{CCNs}$ in South Africa emerged: Poor recognition and diagnosis, inappropriate transport decisions, inefficiencies at patient triage, and delays. These barriers were universally highlighted regardless of the setting of the participants. We discuss the barriers in detail next.

\section{Poor recognition and diagnosis}

The first step in accessing the CCN is recognition of symptoms and diagnosis of STEMI. Recognition needs to occur at two levels: patient recognition and recognition and diagnosis of STEMI by the healthcare provider that the patients presents to primarily (the first medical contact (FMC)).

Participants described delays by the patient in seeking care after symptom onset, "The most common reason for delay presentation is misperception of symptoms which leads to delay in seeking care P11, cardiologist)." Delays in seeking care may mean that these patients have established infarcts and won't be eligible for immediate reperfusion, "you will manage heart failure for a while (P9, EP)." This could be related to the fact that patients misperceive their symptoms as not being serious, attributing it to other more benign conditions, "this could maybe just be heartburn (P1, ECP)." Studies from many different settings, ${ }^{27-30}$ including South Africa, ${ }^{6}$ corroborate the notion that misinterpretation of symptoms and attempts at self-medication are the main reasons for delays in seeking healthcare after symptom onset. A further reason for this delay seemed to be related to access to transport, "they can't get there, either taxis are expensive or taxis don't run after hours (P9, EP)," and "access to a vehicle, is almost unheard of (P9, EP)." Furthermore, access to ambulance transport is also not available within the South African setting, "that's mainly related to the fact that most people cannot access timeous EMS services (P11, cardiol,ogist)" and is reflected in the fact that $84 \%$ of patients do not utilise ambulance transport when experiencing symptoms of ACS. ${ }^{6}$ While the statistics quoted are reported from the South African city of Pretoria, national statistics are not available regarding ambulance usage patterns in STEMI in South Africa. There is further likely to be con- 
siderable variation owing to socio-economic status and geographic spread of patients.

Patients of lower socio-economic status and education were found to have significant delays in seeking care, ${ }^{31}$ and reported not knowing the importance of seeking care early. ${ }^{6}$ In order to overcome this delay in seeking help, participants felt that education campaigns should be considered, "people definitely need that education... so that they recognise what a $M I$ is (P8, EP).” Interestingly, basic education was also considered a challenge that prevents patients from accessing care in a timely manner, "basic education just sort of perpetuates a poverty cycle, which perpetuates their poor access to healthcare (P6, ECP)" and it was felt that education regarding risk factors should be taught at a young age in order to prevent development of CVD in the first place, "if educated correctly from a young age... it could be prevented (P1, ECP)."

Notwithstanding presentation to a PCI-capable facility, within the context of ACS, FMC can occur at two points; a local general practitioner or a prehospital provider via EMS. Participants highlighted the importance of the FMC to manage and treat STEMI, "STEMI is not a disease for a cardiologist, it's a disease for first responders to treat (P11, cardiologist)." Unfortunately, an inability to diagnose STEMI was highlighted at both these levels by participants, "most GPs don't know what an ECG is, they don't know how to interpret it (P11, cardiologist)," and "there may be a misconception in the EMS about the system and how to approach these patients (P1, ECP)."

\section{Inappropriate transport decisions}

Guidelines recommend that should a PCI-capable facility be reachable within 120 minutes of FMC, the patient should be transported there preferentially, even if it means bypassing other facilities. ${ }^{3}$ There appears to be a belief within South African EMS, that patients should always be transported to the closest healthcare facility, regardless of the facility's ability to manage the specific patient, "they wouldn't bypass hospital X to go to hospital Y because they have a PCI facility, they will go to hospital X because that's the closest (P1, ECP)." Transporting patients to non-PCI hospitals is associated with significant delays in reperfusion as participants experience challenges in expediting secondary transport to PCI-facilities, "hours and hours of delays, they have to be transferred to the correct hospital (P1, ECP)." The effect of secondary transport on reperfusion time is well described in the literature. ${ }^{3,6,26,32}$ These delays were also associated with increased mortality. ${ }^{3,32}$
Reasons cited by participants for not bypassing non-PCI capable facilities related to funding and medical insurance status, "there are often issues around funding (P11, cardiologist)," distance to PCI-capable facilities, "And access and transport time (P10, ECP)," being turned away at tertiary centres because patients did not go through the appropriate State referral pathways or geographical drainage areas, "that's not our drainage area (P10, ECP)," and finally a lack of appropriate procedures that guide on transport decisions based on the South African healthcare landscape.

\section{Inefficiencies at patient triage}

Once a patient arrives at hospital, there seems to be significant delays in triaging patients, "people are waiting to be triaged (P11, cardiologist)." According to the South African Triage Scale (SATS), patients who present with chest pain are to be categorised as Orange (to be seen within 10 minutes), and to have an immediate 12 lead ECG done at triage. ${ }^{33}$ Participants felt that this does not occur at triage level, "nurse-based triage didn't work. The SATS is dependent on nurses being well-trained and following it (P8, EP)." This has necessitated many to introduce a doctor-based triage system, to have timely STEMI diagnosis. "At triage we have a doctor, and every chest pain patient gets an ECG at triage, that's checked (P7, EP)."

Furthermore, due to the sheer volume of "Orange" patients within the system, some delays may still take place, therefore necessitating doctors to again reprioritise these patients, "On our very busy days there can be up to 40 unseen patients (P8, EP)," and "reprioritise the orange box and see which orange patient is sicker... a chest pain patient will be seen first $(\mathrm{P} 8$, EP)." This may delay STEMI diagnosis and treatment, "first ECG three hours later (P8, EP)." Participants recommended revisiting triage of these patients and to improve education and training regarding triage, "we have to have good triage system, you have to get an ECG as you come in with your chest pain (P7, EP).”

\section{There are considerable delays}

In addition to delays in recognition and diagnosis, transport and triage, numerous other delays were identified by participants within their system: dispatch delays, "I think there is a massive delay on our dispatch side (P4, ALS)," especially with obtaining the patient's address, "conference with the caller to find the address (P4, ALS)." Once the vehicle arrives on scene, there may be scene delays related to diagnosis or treatment "it doesn't have to take 10, 15 minutes to do a 12 lead ECG (P1, ECP)," and "putting up an IV, is it worth that extra time? (P1, ECP)" Other prehospital delays

African Health Sciences Vol 20 Issue 1, March, 2020 
may be associated with traffic "bad weather, bad traffic (P11, cardiologist)" and road condition, "Our roads look [bad] (P4, ALS)."

Once a patient is transported to a hospital, administrative delays frustrate participants, "the other delay is in opening files for patients (P3, RN:EC in Afrikaans)." Further to this, obtaining the thrombolytic medication once diagnosis has been established, could be delayed by procedures within the pharmacy, "we don't keep things like thrombolysis, there is also a delay in actually getting the medication (P5, doctor)."

Finally, mobilising catheterisation suite staff to the hospital might also delay reperfusion by PCI. Considerable variation in these times could be related to time of day, "simply because the patient came yesterday at 16:30 in the afternoon, they only got here much later (P11, cardiologist)," as staff are not on-site 24 hours a day, "you don't have people waiting there for you (P1, ECP)," "they do not provide a 24/7 service, there are no shifts (P11, cardiologist)."

3. What are some suggested facilitators to optimising a coronary care network?

Considering the complex and varied nature of the South African $\mathrm{CCN}$ as described through the perceptions of our sample, characterised by numerous delays at multiple points; perhaps the goal should not be primary percutaneous intervention, but rather rapid and immediate thrombolysis, followed by coronary angiography and stent placement if feasible. Participants supported this recommendation unanimously. "That should ideally be the standard approach within the setting where we are giving lytics in essence, routinely to STEMIs (P11, cardiologist)," "thrombolyse all of them because of that delay (P10, ECP)," "Maybe we should just thrombolyse every single one and get them to PCI ASAP, or when they fail, STAT (P8, EP)," "Maybe we should get to where we say, a STEMI gets thrombolysed. Period (P10, ECP)," "carry on [with thrombolysis], though I have an on-site lab because I know the reality is that I'm not gonna open that artery within 90 minutes (P11, cardiologist)," and "thrombolyse because the system delays are of such a nature, that patients need to be lysed before they get referred to cath lab in most instances (P11, cardiologist)."

It was suggested that this could be facilitated by means of prehospital thrombolysis, that is underpinned by locally appropriate guidelines and metrics, enforced by legislation and monitored through a clinical governance system. Finally, such a system should be improved by means of local data gathered through quality research.

\section{Prehospital Thrombolysis}

Participants felt that for a subset of patients, prehospital thrombolysis would be the best option, "pre-hospital thrombolysis... it's the only way to go in South Africa (P11, cardiologist)," "to reduce STEMI mortality you must have a Nationwide fibrinolytic programme (P11, cardiologist)," and should likely be applied universally because of anticipated system delays.

Current guidelines suggest that prehospital thrombolysis should be started preferentially, and should be initiated within 10 minutes of STEMI diagnosis, if access to PCI cannot be secured within 120 minutes. Hereafter, patients should be transferred to a PCI facility immediately. ${ }^{3}$ These recommendations are supported by the views of our sample, "paramedic should give reperfusion load that patient and then proceed to the nearest PCI capable Centre, always as a default setting (P11, cardiologist)."

A recent Cochrane Review, suggests that prehospital thrombolysis could be of particular benefit in LMICs, where timely access to primary PCI is not possible. ${ }^{34} \mathrm{An}$ other meta-analysis article demonstrates a reduction in all cause mortality when prehospital thrombolysis is selected over in-hospital thrombolysis. ${ }^{35}$

Participants felt that a prehospital thrombolysis programme should be initiated first on a small scale, "a small attempt at doing prehospital lysis (P6, ECP)," within a system that makes use of telemetry, "send telemetry to the cardiologist or the ED (P1, ECP)" and leverages off of relationships between key role-players towards consultation and communication, "a checklist, and consultation, because at least I could have backing (P1, ECP)" and "the relationship and the communication is the thing (P6, ECP)." Participants suggested that one way of achieving communication and consultation, is through setting up a national STEMI helpline, "some kind of a central helpline where you can call (P11, cardiologist)," and "Here's the STEMI hotline! (P9, EP)" This helpline should be staffed by a cardiologist who can review and diagnose ECGs transmitted and provide advice on the best reperfusion strategy for each particular patient.

Finally, such a programme should be informed by local standard operating procedures and guidelines, "I would like an SOP or something in place (P1, ECP)" and backed up by outcome data, "we are using prehospital thrombolysis... what is their outcome (P10, ECP)." These guidelines should be locally appropriate. 


\section{Locally appropriate guidelines}

Participants felt that applying international guidelines to the South African setting was inappropriate, "we are again adopting American guidelines, European Society guidelines. Where PCI is better because they have all the resources (P9, EP)." These guidelines should focus on STEMI diagnosis, "one Consolidated document which has detail in how to actually diagnose, with pictures (P11, cardiologist)," providing "recommendations for prehospital thrombolysis (P6, ECP)," making recommendations on a specific "geographical referral process (P11, cardiologist)," that is locally applicable to the setting in which the healthcare provider works, "what to do in their setting, whatever that setting is, for that specific individual; but based on a predefined protocol (P11, cardiologist)." Finally, participants recommended an additional, simplified document, "just a single thing that you can stick up on the wall (P11, cardiologist)," that provides regional, "nuanced" recommendations and a simplified means of presenting the contra-indications for thrombolysis, "the contraindications to the lytic it's so hard for somebody, how do you remember all of that (P11, cardiologist)." The ultimate aim is therefore to "in a pragmatic way do the best you can in the setting you are (P11, cardiologist)." Local cardiology and emergency medicine societies were mentioned to assist in developing such guidelines.

Furthermore, these recommendations should be enforced by legislation to ensure equal care for all, "need to create a legal framework which enforces that as a law. It must be legislated because that is emergency care and emergency care is a basic right in our bill of rights (P11, cardiologist)."

\section{Improved clinical governance}

It was felt that current CCNs have "literally no oversight $(\mathrm{P} 6$, $\mathrm{ECP})$," and that "A poor clinical governance system which does not encourage it [CCNs] (P6, ECP)." For this reason, such a system should have "an implementation plan with quality checks" where the treatment of "every STEMI patient... needs to go through clinical governance P6, ECP)." It was also recommended that through consensus and based on evidence, we determine reperfusion targets that are realistic within our context, "a number that works for us (P9, EP)." International guidelines support the views of our sample and suggests that CCNs are subjected to quality reviews and audits, based on appropriate guidelines and quality metrics. ${ }^{3}$ Such quality audits have been associated with widespread system improvements. ${ }^{3}$

\section{Research}

There is a paucity of data regarding cardiovascular dis- ease and STEMI in sub-Saharan Africa, including South Africa. ${ }^{19}$ This need for future research was echoed by our sample, because "we can't adjust the system based on no data (P1, ECP)." It was recommended that a research agenda related to cardiovascular disease be set, a "research team" be established to focus on these aspects towards improvement of our evidence base.

\section{Strengths and limitations}

To our knowledge, this is the first instance where the perceived barriers and facilitators to the optimisation of CCNs in the South African setting was described. A heterogenous sample that had private and state representation, across five of the nine South African provinces and representation from prehospital and in-hospital emergency care and cardiology is a major strength of this study. The study is limited by the number of participants interviewed from each of these groupings, thus limiting the generalisability of the results. As this is a descriptive study, that provides initial exploratory data it is however, not our intent to generalise. A further limitation is the use of a single coder however, this was addressed through interrogating the interpretation through literature published previously, triangulation, reflective admission and through co-author checks of all codes. We recommend further qualitative studies that seek the barriers and facilitators to CCN optimisation from the views of hospital management and support staff, and patients and with larger sample sizes.

We further recommend that further studies seek to explore the effects of these barriers quantitatively.

\section{Conclusion}

The South African coronary care network is perceived be to fragmented and under-resourced, not prioritised by policy-makers and characterised by substantial variation. Barriers to optimised CCNs were related to many system delays throughout the continuum of care.

To overcome these barriers, it was felt that the focus should rather be on timeous thrombolysis for all STEMI patients, that is underpinned by locally applicable guidelines, supported by clinical governance and quality metrics and enforced by legislation. Finally, a paucity of research was highlighted.

\section{Conflict of interest}

None declared. 


\section{References}

1. Onen CL. Epidemiology of ischaemic heart disease in sub-Saharan Africa : review article. Cardiovasc J Afr. 2013;24(2):34-42. doi:10.5830/CVJA-2012-071

2. De Luca G, Suryapranata H, Ottervanger JP, Antman EM. Time Delay to Treatment and Mortality in Primary Angioplasty for Acute Myocardial Infarction: Every Minute of Delay Counts. Circulation. 2004;109(10):1223-1225. doi:10.1161/01.CIR.0000121424.76486.20

3. Ibanez B, James S, Agewall S, et al. 2017 ESC Guidelines for the management of acute myocardial infarction in patients presenting with ST-segment elevation. Eur Heart J. 2018;39(2):119-177. doi:10.1093/eurheartj/ ehx393

4. Schamroth C. Management of acute coronary syndrome in South Africa: insights from the ACCESS (Acute Coronary Events - a Multinational Survey of Current Management Strategies) registry : cardiovascular topics. Cardiovasc J Afr. 2012;23(7):365-370. doi:10.5830/ CVJA-2012-017

5. Maharaj RC, Geduld H, Wallis L a. Door-to-needle time for administration of fibrinolytics in acute myocardial infarction in Cape Town. S Afr Med J. 2012;102(4):241244. http://www.ncbi.nlm.nih.gov/pubmed/22464507.

6. Meel R, Gonçalves R. Time to fibrinolytics for acute myocardial infarction: Reasons for delays at Steve Biko academic hospital, Pretoria, South Africa. South African Med J. 2016;106(1):92-96. doi:10.7196/SAMJ.2016. v106i1.9801

7. Stassen W, Wallis L, Lambert C, Castren M, Kurland L. Percutaneous coronary intervention still not accessible for many South Africans. African J Emerg Med. 2017;7(3):105107. doi:10.1016/j.afjem.2017.04.009

8. Booth A, Hannes K, Harden A, Noyes J, Harris J, Tong A. COREQ (Consolidated Criteria for Reporting Qualitative Studies). In: Guidelines for Reporting Health Research: A User's Manual. Oxford, UK: John Wiley \& Sons, Ltd; 2014:214-226. doi:10.1002/9781118715598. $\operatorname{ch} 21$

9. Holloway I. Basic Concepts for Qualitative Research. Blackwell Science; 1997.

10. Curry LA, Nembhard IM, Bradley EH. Qualitative and mixed methods provide unique contributions to outcomes research. Circulation. 2009;119(10):1442-1452. doi:10.1161/CIRCULATIONAHA.107.742775

11. Shenton AK. Strategies for ensurStrategies for ensuring trustworthiness in qualitative research projects. Educ Inf. 2004;22(2):63-75. http:/ /www.lhemoodle.ch/course/ view.php?id=3229.

African Health Sciences Vol 20 Issue 1, March, 2020
12. Saunders B, Sim J, Kingstone T, et al. Saturation in qualitative research: exploring its conceptualization and operationalization. Qual Quant. 2018;52(4):1893-1907. doi:10.1007/s11135-017-0574-8

13. Erlingsson C, Brysiewicz P. A hands-on guide to doing content analysis. African J Emerg Med. 2017;7(3):93-99. doi:10.1016/j.afjem.2017.08.001

14. Hsieh H-F, Shannon SE. Three Approaches to Qualitative Content Analysis. 2005. doi: $10.1177 / 1049732305276687$

15. Vaismoradi M, Turunen H, Bondas T. Content analysis and thematic analysis: Implications for conducting a qualitative descriptive study. Nurs Health Sci. 2013;15(3):398405. doi:10.1111/nhs.12048

16. Elo S, Kyngäs H. The qualitative content analysis process. J Adv Nurs. 2008;62(1):107-115. doi:10.1111/ j.1365-2648.2007.04569.x

17. Mbewu A. The burden of cardiovascular disease in Sub-Saharan Africa. SA Hear. 2009;6(5):4-10.

18. Ranchod S, Adams C, Burger R, et al. South Africa's hospital sector: old divisions and new developments. In: A P, P B, eds. 2017 SAHR - 20 Year Anniversary Edition. 20th ed. ; 2017:101-109. http://www.hst.org.za/publications/South African Health Reviews/10_South Africas hospital sector_old divisions and new developments.pdf. 19. Hertz JT, Reardon JM, Rodrigues CG, et al. Acute myocardial infarction in sub-Saharan Africa: The need for data. Miranda JJ, ed. PLoS One. 2014;9(5):e96688. doi:10.1371/journal.pone.0096688

20. Sorita A, Ahmed A, Starr SR, et al. Off-hour presentation and outcomes in patients with acute myocardial infarction: systematic review and meta-analysis. BMJ. 2014;348(jan21 4):f7393-f7393. doi:10.1136/bmj.f7393

21. Harris B, Goudge J, Ataguba JE, et al. Inequities in access to health care in South Africa. J Public Health Policy. 2011;32(S1):S102-S123. doi:10.1057/jphp.2011.35

22. Ataguba JE, Akazili J, McIntyre D. Socioeconomic-related health inequality in South Africa: Evidence from General Household Surveys. Int J Equity Health. 2011;10(1):48. doi:10.1186/1475-9276-10-48

23. Kramer E. 'No one may be refused emergency medical treatment' - ethical dilemmas in South African emergency medicine. South African J Bioeth Law. 2008;1(2):5356.

24. Astarcioglu MA, Sen T, Kilit C, et al. Time-to-reperfusion in STEMI undergoing interhospital transfer using smartphone and WhatsApp messenger. Am J Emerg Med. 2015;33(10):1382-1384. doi:10.1016/j.ajem.2015.07.029

25. Meents E, Boyles T. Emergency medical services- 
-poor response time in the rural Eastern Cape. $S$ Afr Med J. 2010;100(12):790. http://www.ncbi.nlm.nih.gov/ pubmed/21414262.

26. Snyders A, Delport R. Referral pathways for reperfusion of STEMI - developing strategies for appropriate intervention. SA Hear Assoc. 2015;12(2):74-80.

27. Khan A, Phadke M, Lokhandwala YY, Nathani PJ. A study of prehospital delay patterns in acute myocardial infarction in an urban tertiary care institute in Mumbai. $J$ Assoc Physicians India. 2017;65(MAY):24-27.

28. George L, Ramamoorthy L, Satheesh S, Saya R, Subrahmanyam DKS. Prehospital delay and time to reperfusion therapy in ST elevation myocardial infarction. $J$ Emerg Trauma Shock. 2017;10(2):64. doi:10.4103/09742700.201580

29. Chai LS, Putit Z, Siop S. Barriers to timely treatment-seeking in patients with acute myocardial infarction in Malaysia: A qualitative study. BMC Nurs. 2016;15(1):33. doi:10.1186/S12912-016-0155-5

30. Taghaddosi M, Dianati M, Fath Gharib Bidgoli J, Bahonaran J. Delay and its related factors in seeking treatment in patients with acute myocardial infarction. ARYA Atheroscler. 2010;6(1):35-41. http://www.pubmedcentral.nih.gov/articlerender.fcgi?artid $=3347806 \&$ tool $=$ pmcentrez\&rendertype $=$ abstract.
31. Albrahim M, Ahmed AM, Alwakeel A, Hijji F, Al-Mallah $\mathrm{MH}$. Predictors of delayed pre-hospital presentation among patients with ST-segment elevation myocardial infarction. Qatar Med J. 2016;2016(1):7. doi:10.5339/ qmj. 2016.7

32. Van Diepen S, Widimský P, Lopes RD, et al. Transfer times and outcomes in patients with ST-segment-elevation myocardial infarction undergoing interhospital transfer for primary percutaneous coronary intervention APEX-AMI insights. Circ Cardiovasc Qual Outcomes. 2012;5(4):437-444. doi:10.1161/CIRCOUTCOMES.112.965160

33. Westen Cape Department of Health. South African Triage Scale: Training Manual.; 2012.

34. M. M, A. L. Pre-hospital versus in-hospital thrombolysis for ST-elevation myocardial infarction. In: McCaul M, ed. Cochrane Database of Systematic Reviews. Vol 2014. Chichester, UK: John Wiley \& Sons, Ltd; 2014:CD010191. doi:http://dx.doi.org/10.1002/14651858.CD010191. pub2

35. Morrison LJ, Verbeek PR, McDonald AC, Sawadsky B V, Cook DJ. Mortality and prehospital thrombolysis for acute myocardial infarction. JAMA. 2000;283(20):26862692. doi:10.1001/jama.283.20.2686 